\title{
Coherent Synthesis of ultra-broadband Optical Parametric Amplifiers
}

\author{
C. Manzoni ${ }^{1}$, S.W. Huang ${ }^{2}$, G. Cirmi ${ }^{3}$, P. Farinello ${ }^{1}$, J. Moses $^{2}$, S. De Silvestri ${ }^{1}$, F. X. Kärtner ${ }^{2,3}$, and \\ G. Cerullo ${ }^{1}$
}

${ }^{1}$ IFN-CNR, Dipartimento di Fisica, Politecnico di Milano, Piazza L. Da Vinci 32, 20133 Milano, Italy

${ }^{2}$ Department of Electrical Engineering and Computer Science and Research Laboratory of Electronics, Massachusetts Institute of Technology, Cambridge, Massachusetts 02139, USA

${ }^{3}$ Center for Free-Electron Laser Science, DESY and University of Hamburg, Notkestraße 85, D22607Hamburg, Germany

\begin{abstract}
We report on coherent synthesis of outputs from two ultra-broadband optical parametric amplifiers; their timing is locked to sub-30-as by a balanced cross-correlator. Synthesised pulses have octave-spanning $(500-1000 \mathrm{~nm})$ spectra and nearly single-cycle 3.8 -fs duration.
\end{abstract}

The generation of sub-single-cycle, carrier-envelope phase (CEP) stable light pulses is one of the frontiers of ultrafast optics, promising to break tremendous new ground in the control of strong-field processes. Shaping the strong electric-field waveform of these pulses can allow steering of ionized electron wavepackets and precise control of tunneling and multiphoton ionization events. The two key ingredients for sub-single-cycle pulse generation are ultrabroad bandwidths, exceeding one octave, and accurate control of the spectral phase, so as to obtain nearly transform-limited (TL) pulsewidths. These requirements are very challenging to satisfy with a single laser source, and thus intense research activity is devoted to the coherent synthesis of pulses generated by separate sources. Coherent addition of two pulse trains derived from the same fiber laser resulted in the first demonstration of an isolated single-cycle optical pulse [1], although with low (nJ-level) energy. SPM in a hollow-core fiber, followed by dispersion management of different portions of the ultrabroadband spectrum and coherent superposition of the compressed sub-pulses, has recently allowed the generation of sub-cycle light pulses with hundreds of $\mu \mathrm{Js}$ energy [2]. However, further energy scaling is limited by the handling capabilities of the fiber. Coherent combination of the outputs from ultra-broadband optical parametric amplifiers (OPAs) or optical parametric chirped pulse amplifiers is an alternative and promising route, with the advantages of generating smooth spectra and straightforward energy scaling [3].

Here we demonstrate coherent synthesis of the outputs from two ultra-broadband OPAs pumped by a Ti:sapphire laser and seeded by the same white-light continuum (WLC). Broadband gain in an OPA is achieved either employing a degenerate OPA (DOPA), or in the non-collinear OPA (NOPA). Using these configurations, a variety of OPA schemes, pumped by either the fundamental frequency (FF) or the second harmonic (SH) of Ti:Sapphire, have been demonstrated. In particular, 5-fs visible 
pulses (500-700 nm) are routinely generated by SH-pumped NOPAs, and sub-7-fs pulses have been produced in the $650-950 \mathrm{~nm}$ spectral region by the SH-pumped DOPA [4]. Here we demonstrate coherent synthesis of the NOPA and the DOPA output, resulting in octave-spanning (520-1000 nm) spectra supporting nearly single-cycle sub-4-fs-pulses.

(a)

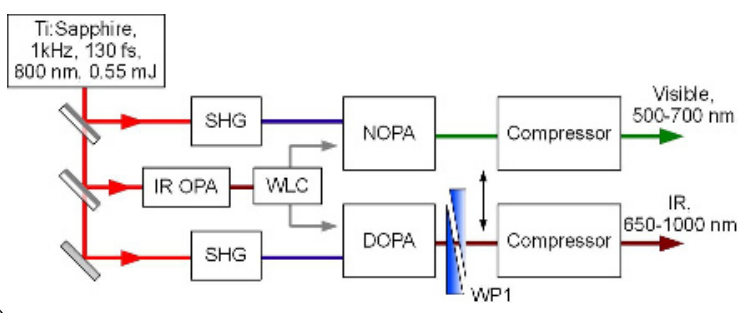

(b)

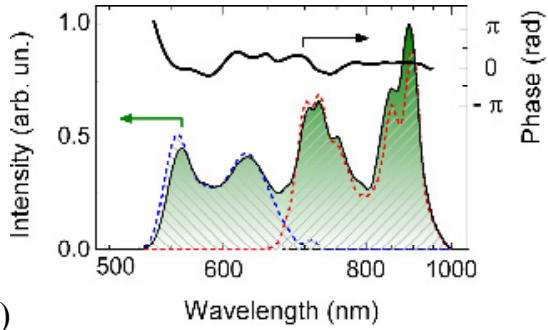

Fig. 1: (a) setup for separate amplification, compression and combination of visible and near-infrared pulses. SHG: second harmonic generation; WLC: white light continuum generation; WP1: wedge plates for the control of relative phase. (b) Amplified spectra of the OPAs (dashed lines) and of the synthesized pulses, supporting 3.6 fs pulse duration. Top panel reports the spectral phase retrieved from 2DSI measurements.

The experimental setup for coherent synthesis of OPAs, summarized in the block diagram of Fig. 1, consists of (i) the generation of a broadband WLC seed; (ii) two broadband OPAs seeded by distinct portions of the WLC; (iii) coherent combination of their outputs in order to synthesize (sub-) singlecycle pulses. The system is powered by $550-\mu \mathrm{J}$ pulses from an amplified, Ti:sapphire laser, providing $130-\mathrm{fs}$ pulses at $800-\mathrm{nm}$ wavelength and $1-\mathrm{kHz}$ repetition rate. Since the WLC presents a highly structured intensity and phase profile around the driving pulse wavelength, it is not possible to use the WLC directly generated by the FF in order to seed the DOPA, and an additional stage is necessary to shift the WLC driving wavelength to the infrared (IR), thus generating a well-behaved WLC around $800 \mathrm{~nm}$. The two-stage near-IR OPA is pumped by the FF of Ti:sapphire and seeded by the WLC generated in a sapphire plate. When pumped by $250-\mu \mathrm{J}$ pulses, this OPA provides a total signal + idler energy of $35 \mu \mathrm{J}$. In addition, the difference frequency generation process occurring between pump and signal sharing the same CEP produces idler pulses with passively stabilized CEP [5]. Both the signal and the CEP-stable idler can generate a broadband spectrally well-behaved WLC, either in sapphire or YAG plates.

The short-wavelength portion of this seed ranges from 500 to $1000 \mathrm{~nm}$, and covers without gaps the amplification range of the subsequent visible NOPA and SH-pumped DOPA. The seed is then split by a neutral-density ultrathin beam-splitter, and the two replicas are amplified by the visible NOPA and by the DOPA. When pumped by the SH from the remaining $300-\mu \mathrm{J} F F$ pulses, each of the two OPAs provides pulses with an energy of 1-2 $\mu \mathrm{J}$. Compression of the two outputs is achieved by visible and IR chirped mirrors, which provide pulsewidths close to the TL values $(\approx 6$ fs for the visible NOPA and $\approx 8$ fs for the DOPA). The two amplified pulses are then synchronized by a delay line equipped with a piezoelectric actuator, and collinearly combined by a second ultrathin beamsplitter. A gap-free spectrum arising from the combination of the two pulses is shown in Fig. 1(b); it supports a TL 3.6-fs pulse duration.

The last step of the pulse synthesis is the coherent combination of the two pulses, which calls for careful control of their relative delay and CEP. The delay can be finely tuned by the piezoelectric actuator, the relative CEP by wedge pair WP1 inserted in the path of the DOPA beam. The relative delay of the pulses was detected by a balanced optical cross-correlator (BOC) [6], which consists of two identical cross-correlators using 1-mm-thick BBO crystals, phase matched for sum-frequency generation of $600-\mathrm{nm}$ light from the NOPA and $800-\mathrm{nm}$ light from the DOPA. By canceling the amplitude noise, the BOC allows attosecond-precision relative timing measurement. The low-pass filtered signal from the BOC was fed to a PID controller driving the piezo translator. The closed loop allowed suppression of relative delay fluctuations to below 30 -as over 10 minutes, corresponding to less than $1 / 80^{\text {th }}$ optical cycle (Fig. 2(a)). 

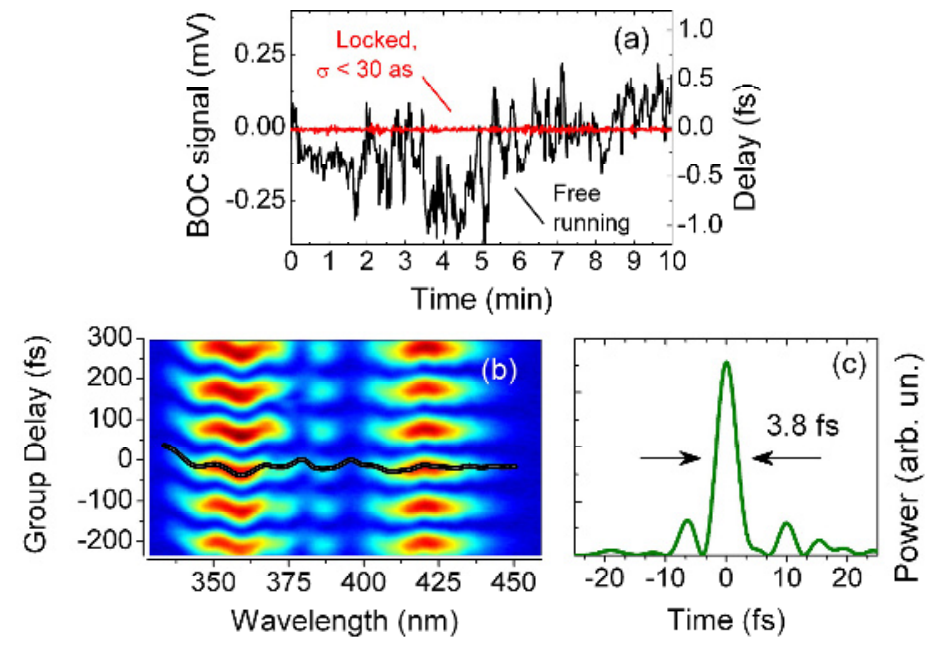

Fig.2: (a) Signal from the BOC and corresponding pulse delay. Black line: free-run operation of the system; red line: pulse delay is locked by the feedback. (b) 2DSI map and retrieved group delay; the signal arises from sumfrequency generation of the whole pulse with components at $900 \mathrm{~nm}$. (c) Intensity of the reconstructed pulse.

The last remaining experimental challenge is the full temporal characterization of the synthesized ultra-broadband pulse, which has been performed by two-dimensional spectral-shearing interferometry (2DSI) [7]. Figure 2(b) shows a 2DSI map and the corresponding retrieved frequencydependent group delay (GD), which is substantially flat over the whole pulse bandwidth; the residual GD oscillations in the visible spectral range are due to the large number of bounces on the chirped mirrors.

The retrieved spectral phase, reported in Fig. 1(b), was applied to the measured spectral amplitude, giving the pulse shown in Fig. 2(c). The FWHM of its intensity profile is 3.8 fs, indicating that the pulse is compressed to within $4 \%$ of the TL duration. The oscillation of the electric field in the side lobes is small enough to have negligible contribution to strong field processes, like CEP sensitive field emission

This proof-of-principle experiment works with limited available pump energy, however scaling of the energy of both OPAs to the mJ-level by addition of power amplification stages should be straightforward. In the future, further wavelength multiplexing by the coherent addition of other ultra-broadband OPAs [8] should allow the generation of high-quality sub-cycle pulses.

\section{References}

[1] G. Krauss, S. Lohss, T. Hanke, A. Sell, S. Eggert, R. Huber, and A. Leitenstorfer, Nature Photonics 4, 33 (2010).

[2] A. Wirth, M. Th. Hassan, I. Grguraš, J. Gagnon, A. Moulet, T. T. Luu, S. Pabst, R. Santra, Z. A. Alahmed, A. M. Azzeer, V. S. Yakovlev, V. Pervak, F. Krausz, and E. Goulielmakis, Science 334, 195 (2011).

[3] S.-W. Huang, G. Cirmi, J. Moses, K.-H.Hong, S. Bhardwaj, J. R. Birge, L.-J. Chen, E. Li, B. J. Eggleton, G. Cerullo and F. X. Kärtner, Nature Photonics 5, 475 (2011).

[4] A. M. Siddiqui, G. Cirmi, D. Brida, F. X. Kärtner, and G. Cerullo, Opt. Lett. 34, 3592 (2009).

[5] A. Baltuška, T. Fuji, and T. Kobayashi, Phys. Rev. Lett. 88, 133901 (2002).

[6] T. R. Schibli, J. Kim, O. Kuzucu, J. T. Gopinath, S. N. Tandon, G. S. Petrich, L. A. Kolodziejski, J. G. Fujimoto, E. P. Ippen, and F. X. Kaertner, Opt. Lett. 28, 947 (2003).

[7] J. R. Birge, H. M. Crespo, and F. X. Kärtner, J. Opt. Soc. Am. B 27, 1165 (2010).

[8] D. Brida, C. Manzoni, G. Cirmi, M. Marangoni, S. Bonora, P. Villoresi, S. De Silvestri, and G. Cerullo, J. Opt. 12, 013001 (2010). 